\title{
Résultats d'enquête sur les problèmes posés par la fabrication du yaourt en République Populaire du Congo
}

\author{
par \\ M. J. PEYROT* et J.P. LARPENT*
}

Si la technique de fabrication du yaourt et les méthodes de contrôle sont bien connues en France [1, 2, 3, 4, 5] et dans beaucoup de pays producteurs $[6,7,8,9,10,12]$ de climat tempéré, peu de recherches ont été effectuées et les résultats publiés pour améliorer la production dans les pays en voie de développement [11] dont les conditions climatiques imposent des contraintes fort différentes.

L'emploi systématique de poudres de lait peut également modifier les qualités de la matière première. Nous avons eu la possibilité d'apprécier les problèmes posés par ce type de produit en République Populaire du Congo. Cet exemple doit permettre la généralisation des conclusions aux pays de climat tropical ou équatorial. Nous espérons contribuer au développement de ces fabrications car le yaourt gagne peu à peu une certaine popularité dans les pays en voie de développement pour diverses raisons :

- c'est un produit réalisable, sans beaucoup de difficulté, à partir de lait sec ;

- il ne provoque pas d'allergie au lactose comme le lait ;

- sa valeur nutritionnelle est reconnue et il apporte du calcium ;

- c'est un produit rafraîchissant relativement facile à fabriquer artisanalement.

A Brazzaville, les yaourts sont faits à partir de lait reconstitué à des taux variables selon les fabricants (15-16 p.100: marque A ; 17-18 p. 100 : marque B ; 15 p. 100 : marque C). L'eau utilisée est l'eau de distribution de la ville le plus souvent non filtrée. Les sacs de poudre de lait sont "gardés » à la température ambiante et utilisés dans les $24 \mathrm{~h}$ suivant leur ouverture.

\footnotetext{
* Laboratoire de Microbiologie, 4, rue Ledru - 63000 Clermont-Ferrand.
} 
Le lait est amené seulement pendant $30 \mathrm{~s}$ (maximum) à $70^{\circ} \mathrm{C}$. Les levains, sous forme lyophilisé, sont conservés à $+5^{\circ} \mathrm{C}$ environ. Chaque jour des yaourts de la veille sont utilisés comme ferments à raison de 42 pots de $125 \mathrm{ml}$ pour 801 de lait reconstitué. L'opération d'ensemencement n'est pas pratiquée stérilement. En fin de fabrication les yaourts atteignent en moyenne $75^{\circ} \mathrm{D}$.

\section{MATERIEL ET METHODES}

Quatre types de yaourts à raison d'un minimum de quinze échantillons différents par marque ont été étudiés à Brazzaville. Les milieux de culture utilisés sont ceux préconisés pour les contrôles dans les industries agricoles et alimentaires: Plate Count agar pour la flore totale mésophile, la gélose au désoxycholate lactose pour les coliformes, le milieu de Baird Parker pour les staphylocoques avec test coagulase ultérieur, la gélose TSN pour les anaérobies sulfitoréducteurs. Les germes aérobies at aérobies-anaérobies facultatifs thermo-résistants sont recherchés sur gélose FIL après chauffage $30 \mathrm{~min}$ à $63^{\circ} \mathrm{C}$.

Les levures et les moisissures sont isolées sur milieu OGA, les bactéries lactiques sur MRS à $\mathrm{pH} 5,4$ ou dans le cas de S. thermophilus sur milieu M17 ou milieu d'Elliker.

Pour tenter de dégager les exigences du consommateur congolais nous avons réalisé plusieurs dégustations et tests organoleptiques avec un jury composé de six Congolais et de six Français. La technique utilisée est celle des préférences par la méthode des rangs. Un yaourt d'importation (D) et un fabriqué au laboratoire (E) ont été ajoutés aux trois de marques locales différentes. Les questionnaires ont porté sur l'apparence (notée 3 points), le goût (doux, ou aigre noté sur 10 points) et la consistance (cassant, moelleux, ferme, synérèse notée sur 10 points). Les moyennes obtenues ont permis d'obtenir un classement entre les cinq types de yaourts.

\section{RESULTATS}

\section{Contrôle des matières premières}

Sept lots de poudre ont été contrôlés. Des tests de conservation ont été réalisés à $5^{\circ} \mathrm{C}$ et $25^{\circ} \mathrm{C}$ pendant $31 \mathrm{j}$ et les contrôles microbiologiques au $1^{\mathrm{er}}, 5^{\mathrm{e}}, 10^{\mathrm{e}}, 15^{\mathrm{e}}, 25^{\mathrm{e}}$ et $31^{\mathrm{e}}$ jour. Quantitativement les normes des fabricants de poudre de lait sont bien respectées dans l'ensemble. 
TABLEAU 1

Contrôle de divers lots de poudres de lait (résultats donnés/ml)

\begin{tabular}{|c|c|c|c|}
\hline Type de micro-organismes & Lait entier & Lait écrémé & Normes \\
\hline Aérobies mésophiles $32^{\circ} \mathrm{C}-72 \mathrm{~h}$ & $8.10^{3}-5,9.10^{6}$ & $6.10^{2}-9,7.10^{3}$ & $<2.10^{5}$ \\
\hline Thermorésistants & $3,4 \cdot 10^{3}-4,8 \cdot 10^{3}$ & $1,6.10^{2}-4,8.10^{3}$ & \\
\hline Coliformes totaux & 0 & 0 & $<25$ \\
\hline Coliformes fécaux & 0 & 0 & 0 \\
\hline Streptocoques fécaux & $0-6$ & 0 & 0 \\
\hline Anaérobies sulfito-réducteurs & 0 & 0 & 0 \\
\hline Levures et moisissures & $4,2.10^{1}$ & $5.10^{2}$ & $<10^{2}$ \\
\hline \multicolumn{4}{|l|}{$\begin{array}{l}\text { Caractéristiques physico- } \\
\text { chimiques : }\end{array}$} \\
\hline Acidité Dornic & $13-15^{\circ} \mathrm{D}$ & $15,8-18,5^{\circ} \mathrm{D}$ & $15^{\circ} \mathrm{D}$ \\
\hline Humidité en p. 100 & $3,03-5,7$ & $4,02-4,55$ & $<4$ p. 100 \\
\hline Solubilité & 97,6 & $99,3-99,5$ & $98<\mathrm{S}<100$ \\
\hline Amidon & absence & absence & absence \\
\hline
\end{tabular}

Cependant les levures et les moisissures sont trop souvent présentes et les germes totaux comprennent beaucoup de bactéries thermorésistantes (tab. 1). L'absence de cahier des charges avec le fabricant et la conservation des lots sans précaution expliquent ces résultats. Les difficultés apparaissent rapidement puisque le traitement thermique imposé au lait reconstitué n'est, dans notre cas, que de $30 \mathrm{~s}$ à $70^{\circ} \mathrm{C}$, ce qui est notoirement insuffisant.

L'eau de reconstitution (six échantillons) pose de graves problèmes, les matières en suspension forment des dépôts rougeâtres dans les récipients. Ses qualités hygiéniques sont médiocres : présence fréquente suivant les échantillons d'Escherichia coli $(2 / \mathrm{ml})$, de staphylocoques pathogènes $(12 / \mathrm{ml})$. Les germes aérobies mésophiles dépassent souvent $4.10^{3} / \mathrm{ml}$.

Les levains ne sont pas entretenus stérilement et chaque jour des yaourts de la veille sont utilisés comme ferment (ensemencement à $6,5 \mathrm{p} .100$ ), ceci pendant 18 à $30 \mathrm{j}$. Le levain est alors totalement déséquilibré, les yaourts sont très acides et peu aromatiques (tab. 2). 


\section{Analyse du produit fini}

Les résultats obtenus pour les trois marques de yaourt sont résumés dans le tableau 3. Les coliformes sont souvent présents en

\section{TABLEAU 2}

Evolution des populations de Lactobacillus bulgaricus et de Streptococcus thermophilus au cours des fabrications

\begin{tabular}{|c|c|c|c|c|c|c|c|c|}
\hline \multirow[b]{2}{*}{ Jours } & \multicolumn{4}{|c|}{ Yaourt nature } & \multicolumn{4}{|c|}{ Yaourt aromatisé } \\
\hline & 1 & 4 & 8 & 12 & 1 & 4 & 8 & 12 \\
\hline Lactobacillus & $9,7.10^{9}$ & $3,3.10^{9}$ & $3,6.10^{9}$ & $1,5.10^{11}$ & $4,8.10^{9}$ & $2,2.10^{9}$ & $4,5.1010$ & $2,4.10^{10}$ \\
\hline Streptococcus & $2,6.10^{8}$ & $9.10^{8}$ & $6,8.108$ & $1,1.10^{8}$ & $4.10^{9}$ & $1,1.10^{9}$ & $5,6.10^{9}$. & 1010 \\
\hline$S / L$ & 0,027 & 0,27 & 0,19 & 0,0007 & 0,83 & 0,5 & 0,12 & 0,42 \\
\hline $\mathrm{L}=$ Lactoba & \multicolumn{8}{|c|}{$\mathrm{S}=$ Streptococcus. } \\
\hline
\end{tabular}

TABLEAU 3

Analyses de trois types de yaourts

\begin{tabular}{l|c|c|c}
\hline & Marque A & Marque B & Marque C \\
\cline { 2 - 3 } Anaérobies sulfito-réducteurs & 0 & 0 & 0 \\
Coliformes totaux & $0-1,7.10^{3}$ & $0-1,5.10^{3}$ & $0-1,7.10^{2}$ \\
Streptocoques fécaux & $0-1,3.10^{4}$ & $3,6.10^{2}-8,4.10^{3}$ & $2,4.10^{2-10^{3}}$ \\
Micrococcaceae & $0-1,2.10^{4}$ & $2.10^{3}-1,2.10^{4}$ & $0-10^{3}$ \\
Staphylocoques pathogènes & $0-4.10^{2}$ & $4.10^{3}-4,8.10^{4}$ & 10 \\
Levures & $0-1,9.10^{8}$ & $0-3,5.10^{7}$ & $0-6,3.10^{7}$ \\
Moisissures & $0-10^{2}$ & $0-10^{3}$ & $0-10^{2}$ \\
Streptococcus thermophilus & $1,4.10^{6}-1,1.10^{10}$ & $4.10^{7}-1,9.10^{9}$ & $5,2.10^{9}-1,2.10^{10}$ \\
Lactobacillus bulgaricus & $1,4.10^{9}-3,9.10^{10}$ & $1,2.10^{8}-8.10^{9}$ & $8.10^{8}-6.10^{10}$ \\
S/L & $0,0007-3$ & $0,005-1$ & $0,2-1$ \\
\hline
\end{tabular}




\section{TABLEAU 4}

Résultats des deux séries de tests organoleptiques organisés avec jurys de dégustateurs congolais et français

\begin{tabular}{|c|c|c|c|c|c|c|}
\hline \multirow{2}{*}{ Yaourts } & \multicolumn{3}{|c|}{ Première série } & \multicolumn{3}{|c|}{ Deuxième série } \\
\hline & $\mathrm{pH}$ & $\begin{array}{c}\text { Jury } \\
\text { congolais }\end{array}$ & $\begin{array}{l}\text { Jury } \\
\text { français }\end{array}$ & $\mathrm{pH}$ & $\begin{array}{c}\text { Jury } \\
\text { congolais }\end{array}$ & $\begin{array}{c}\text { Jury } \\
\text { français }\end{array}$ \\
\hline A & 3,9 & $5^{\mathrm{me}}$ & $3^{\mathrm{me}}$ & 4,1 & $4^{\mathrm{me}}$ & $4^{\mathrm{me}}$ \\
\hline B & 3,9 & $2^{\text {me }}$ & $1^{\text {er }}$ & 4,1 & $2^{\mathrm{me}}$ & $1^{\text {er }}$ \\
\hline $\mathrm{C}$ & 3,9 & $3^{\mathrm{me}}$ & $2^{\mathrm{me}}$ & 4,1 & $3^{\mathrm{me}}$ & $2^{\mathrm{me}}$ \\
\hline $\begin{array}{c}\mathrm{D} \\
\text { (importation) }\end{array}$ & 4,1 & $4^{\mathrm{me}}$ & $4^{\mathrm{me}}$ & 4,4 & $5^{\mathrm{me}}$ & $5^{\mathrm{me}}$ \\
\hline $\begin{array}{c}\mathrm{E} \\
\text { (témoin laboratoire) }\end{array}$ & 4,2 & $1^{\mathrm{er}}$ & $5^{\mathrm{me}}$ & 4,0 & $1^{\mathrm{er}}$ & $3^{\text {me }}$ \\
\hline
\end{tabular}

grande quantité ainsi que les entérocoques et les staphylocoques pathogènes. Les moisissures parfois très abondantes sont accompagnées de levures dont la multiplication abaisse fortement la durée de vie du produit : les yaourts ont des goûts désagréables (de levure) et des gaz s'accumulent. L'équilibre des ferments lactiques est extrêmement variable et toujours déséquilibré en faveur de Lactobacillus bulgaricus.

\section{Conservation du produit fini}

Après $40 \mathrm{j}$ de conservation à $+10^{\circ} \mathrm{C}$ environ, le nombre de ferments lactiques dépasse encore $10^{8}$. Le $\mathrm{pH}$ et l'acidité titrable varient peu $\left(\mathrm{pH} \# 3,8\right.$ et $\left.80^{\circ} \mathrm{D}\right)$. La durée de vie du yaourt serait normale si trop souvent les contaminations, levures et moisissures, ne venaient la raccourcir en rendant le produit peu attractif (goût et aspect) dès le $10^{\mathrm{e}}$ jour de conservation.

De plus, très souvent, les yaourts ne sont pas conservés, ni livrés dans de bonnes conditions $\left(t=25^{\circ} \mathrm{C}\right)$. Dans ce cas, l'acidité diminue rapidement à cause des levures consommatrices d'acide lactique. La remontée du $\mathrm{pH}$ peut évidemment représenter un danger hygiénique.

Les deux jurys de nationalité différente étaient d'accord pour refuser la marque d'importation : mauvaise conservation, trop forte 
acidité, exsudation de sérum ou consistance trop épaisse. Une marque locale (A) a été également refusée à cause du sérum libre abondant, de sa consistance épaisse pâteuse, sèche et de son acidité trop importante (tab. 4).

Les jugements par contre s'opposent à propros de trois autres marques. Le yaourt E classé cinquième par le jury français était classé premier par les Congolais (goût âpre). Par contre un accord se réalise pour la marque B locale entre les deux jurys. Le caractère âpreté n'est pas suffisamment prononcé pour être refusé par le jury français.

\section{DISCUSSION - CONCLUSIONS}

Si les normes des poudres de lait sont dans l'ensemble respectées la qualité de l'eau de reconstitution laisse beaucoup à désirer. La thermisation $30 \mathrm{~s}$ à $70^{\circ} \mathrm{C}$ est insuffisante, à plus forte raison sous ces climats favorisant le développement des levures et à un moindre degré des moisissures.

Les micro-organismes dangereux (coliformes, staphylocoques pathogènes) sont très souvent présents rendant le produit hygiéniquement inacceptable. Technologiquement les fortes populations de levures diminuent la durée de vie du produit et créent des mauvais goûts.

Il est nécessaire de promouvoir un entretien correct des ferments car le fait de repartir du yaourt de la veille prélevé au hasard pendant plusieurs semaines crée une irrégularité importante des populations de Streptococcus thermophilus et de Lactobacillus bulgaricus.

Si les fabricants veulent étendre leur marché il est indispensable d'allonger la durée de vie de leurs produits : ceci passe en priorité par une amélioration des conditions d'hygiène lors des fabrications, une thermisation suffisante, une fabrication de levains ou à la rigueur un emploi systématique de levains lyophilisés. Un effort plus important devra être réalisé pour contrôler l'eau servant à la reconstitution du lait. Enfin, il faudra qu'ils définissent mieux les exigences des consommateurs. Les quelques essais menés par nous montrent que les critères d'appréciation ne sont pas identiques à ceux définis pour les consommateurs français. Il semble qu'un goût plus acide, plus âpre, satisfasse mieux le consommateur congolais.

\section{Remerciements}

Nous tenons à remercier MM. Accolas (I.N.R.A., Jouy-en-Josas), Balesfontaines (Sté Richesmonts) et Vayssier (Sté Lacto-Labo), pour leurs conseils techniques et leur aide matérielle. 


\section{Ré s u m é}

La fabrication des yaourts en pays à climat tropical ou équatorial pose de nombreux problèmes hygiéniques et technologiques : contrôle et conservation des poudres de lait, établissement d'un barème de pasteurisation correct, utilisation d'une eau potable, entretien des levains. La plus grande difficulté vient du développement des levures. Cette étude souligne l'importance de la définition des caractères organoleptiques exigés par la population locale.

\section{S u m mary}

\section{INVESTIGATION ON YOGHURT FABRICATION IN CONGO REPUBLIC}

The fabrication of yoghurt in tropical or equatorial countries is difficult. The problems are following: controls and conservation of milk powder, temperature of pasteurisation, microbiology of water, culture of starter. The most important difficulty is the yeast growth. This study shows the importance of organoleptic characteristics required by local population.

Reçu pour publication en janvier 1981.

\section{Bibliographie}

[1] Accolas (J. P.), Bloquel (R.), Didienne (R.) et Régnier (J.) (1977). - Propriétés acidifiantes des bactéries lactiques thermophiles en relation avec la fabrication du yaourt. Le Lait, 561-562, 1-23.

[2] Accolas (J. P.) (1979). - Caractéristiques taxonomiques et identification de Lactobacillus bulgaricus et de Streptococcus thermophilus. FIL/IDF, groupe E 44.

[3] Alais (Ch.) (1974). - Sciences du lait, 3e éd., SEP, Paris.

[4] Contrôle de la qualité des produits laitiers $T_{2}$. Analyse microbiologique et analyse sensorielle. Direction des Services Vétérinaires, Ministère de l'Agriculture (France), 1971.

[5] FIL/IDF. - International Standard FIL/IDF 47, 1969, Rpt CE Doc. 15/1967, Brussels.

FIL/IDF. - Milchwiss., 1971, 28, 344.

FIL/IDF. - Michwiss., 1971, 28, 345.

[6] Hammer (B. W.) and Babel (F. J.) (1957). - Dairy bacteriology, 4 th ed., Wiley and sons, New York.

[7] Kosikowski (F. V.) (1966). - Cheese and fermented foods. Edw. Broth. Inc., Michigan.

[8] Lusiani (G.), Salvadori (P.) et Bianchi-Salvadori (B.) (1974). - Evaluations microbiologiques sur le yaourt en rapport avec les temps et les températures de conservation. Le Lait, 531-532, 53-59. 
[9] Rasic (J. L. J.) and Kurmann (J. A.) (1978). - Yoghurt, 428 p. Technical Dairy Publishing House.

[10] Speck (M. L.) (1979). - Yoghurt qualities effected by starters and processing. Dairy Industries International, 5-7.

[11] Veysseire (R.) (1979). - Le lait et les pays en voie de développement. Revue Laitière Française, 381, 19-30.

[12] Walter (J.), Hasselmann (M.) et Castillo (M.) (1978). - Aspects quantitatifs de la flore bactérienne normale des yaourts. Revue Laitière Française, 362, 117-129. 